\title{
An interaction of a sonic injection jet with a supersonic turbulent flow approaching a re-entry vehicle to atmosphere
}

\author{
D. Sun \& R. S. Amano \\ Department of Mechanical Engineering, \\ University of Wisconsin-Milwaukee, USA
}

\begin{abstract}
A shock-interaction flow field generated by transverse sonic injection into a supersonic flow was simulated by solving the Favre-averaged Navier-Stokes equations using the weighted essentially non-oscillatory (WENO) schemes. Three-dimensional results indicate that there appear four pairs of vortices around the secondary injection. In the upstream of the square injection there exist two main recirculation regions and the primary vortex induces the horseshoe vortex region. After the secondary injection flow ejects from the square hole, it is forced by the supersonic main flow and then it becomes a pair of counter rotation vortices towards downstream. In the downstream there is a low-pressure region that conduces a pair of helical vortices.

Keywords: supersonic flow, bow shock wave, horse-shoe vortex, CFD.
\end{abstract}

\section{Introduction}

Shock-interaction flowfield generated from a sonic gaseous flow injected transversely into a supersonic freestream is encountered in practical applications such as space shuttle reentry atmosphere, rocket motor thrust vector control systems, supersonic combustion, high-speed flight vehicle reaction control jets, and gas-turbine cooling systems [1-3]. One example is the case when re-entry vehicles or reusable rockets enter atmosphere, its attitude has to be controlled to endure large aerodynamic heating. Because of a high angle of attack on re-entering, the flow separates from the control surface. If the jet is injected into a hypersonic flow, complicated interaction between the jet and the flow occurs. It results in boundary layer separation, shock waves, and vortices, which are 
schematically shown in Fig. 1. The mixing flow field is very complex, which includes a bow shock wave in front of the injection, boundary layer separation and vortices. Therefore a higher-order scheme is needed when computing such a flow field. In 1980s, a new high-order scheme, essentially non-oscillatory scheme (ENO), was introduced by Harten et al. [4] Later, Wilcoxson and Manousiouthakis [5] and Jiang and Shu [6] developed the weighted ENO schemes based on ENO schemes, which are simpler and more efficient.

In 3-D flowfield, a three-dimensional bow shock forms ahead of the injection and interacts with the approaching boundary layer, resulting in a separation bubble. A barrel shock also occurs as the under expanded jet accelerates into the free stream. Acceleration of the jet core flow continues until a normal shock, or Mach disk, forms. Directly downstream of the jet plume another separated zone develops in the region between the jet exit and the boundary layer reattachment point. A pair of counter-rotating vortices generated within the jet fluid and a horseshoe vortex region also forms near the jet exit and wraps around the injector as illustrated in the schematic.

In this paper, the fifth-order WENO scheme of Jiang and Shu [6] and the $k-\varepsilon$ turbulent model are used to calculate the supersonic flowfield with secondary injection. The freestream Mach number is 3.7 and 3.0 in two and threedimensional flowfield, respectively, and the injections in both flowfield are sonic. The slot width of the injection in two-dimensional flowfield is $1 \mathrm{~mm}$ and the orific of the injection in three-dimensional flow is a cubic whose width is $1 \mathrm{~mm}$. The conditions of the two-dimensional flowfield is the same with reference 4.

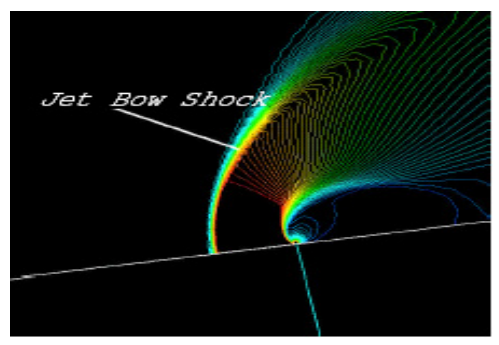

Figure 1: $\quad$ Bow shock in a mixing jet-stream.

The schematic of the transverse jet injected into a supersonic cross flow is shown in Fig. 1. A three-dimensional bow shock is formed ahead of the injected stream and it interacts with the approaching boundary layer, resulting in a separation bubble. A barrel shock also occurs as the under-expanded jet accelerates into the cross flow. Acceleration of the jet core flow continues until a normal shock, or Mach disk, forms. Directly downstream of the jet plume another separated zone develops in the region between the jet exit and the boundary layer reattachment point. A pair of counter-rotating vortices generated within the jet fluid and a horseshoe vortex region also forms near the jet exit and wraps around the injector as illustrated in the schematic. 


\section{Governing equations}

The three-dimensional Favre-averaged Navier-Stokes equations and the low-Reynolds number $k-\varepsilon$ turbulent model are given as follows:

$$
\frac{\partial Q}{\partial t}+\frac{\partial}{\partial x}\left(E-E_{v}\right)+\frac{\partial}{\partial y}\left(F-F_{v}\right)+\frac{\partial}{\partial z}\left(G-G_{v}\right)=H
$$

$$
Q=\left(\begin{array}{c}
\rho \\
\rho u \\
\rho v \\
\rho w \\
e \\
\rho k \\
\rho \varepsilon
\end{array}\right)
$$

$$
E=\left(\begin{array}{c}
\rho u \\
\rho u^{2}+p \\
\rho u v \\
\rho u w \\
u(e+p) \\
\rho u k \\
\rho u \varepsilon
\end{array}\right)
$$

$$
F=\left(\begin{array}{c}
\rho v \\
\rho u v \\
\rho v^{2}+p \\
\rho v w \\
v(e+p) \\
\rho v k \\
\rho v \varepsilon
\end{array}\right)
$$

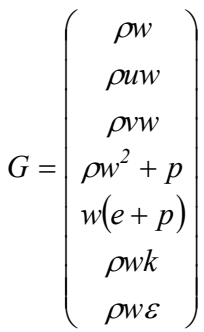

$$
E_{v}=\left(\begin{array}{c}
0 \\
\tau_{x x} \\
\tau_{x y} \\
\tau_{x z} \\
u \tau_{x x}+v \tau_{x y}+w \tau_{x z}-q_{x} \\
\left(\mu+\frac{\mu_{t}}{\sigma_{k}}\right) \frac{\partial k}{\partial x} \\
\left(\mu+\frac{\mu_{t}}{\sigma_{\varepsilon}}\right) \frac{\partial \varepsilon}{\partial x}
\end{array}\right)
$$

$$
F_{v}=\left(\begin{array}{c}
0 \\
\tau_{x y} \\
\tau_{y y} \\
\tau_{y z} \\
u \tau_{x y}+v \tau_{y y}+w \tau_{y z}-q_{y} \\
\left(\mu+\frac{\mu_{t}}{\sigma_{k}}\right) \frac{\partial k}{\partial y} \\
\left(\mu+\frac{\mu_{t}}{\sigma_{\varepsilon}}\right) \frac{\partial \varepsilon}{\partial y}
\end{array}\right)
$$

$$
G_{v}=\left(\begin{array}{c}
0 \\
\tau_{x z} \\
\tau_{y z} \\
\tau_{z z} \\
u \tau_{x z}+v \tau_{y z}+w \tau_{z z}-q_{z} \\
\left(\mu+\frac{\mu_{t}}{\sigma_{k}}\right) \frac{\partial k}{\partial z} \\
\left(\mu+\frac{\mu_{t}}{\sigma_{\varepsilon}}\right) \frac{\partial \varepsilon}{\partial z}
\end{array}\right) H=\left(\begin{array}{c}
0 \\
0 \\
0 \\
0 \\
0 \\
\mu_{t} P-\rho \varepsilon \\
c_{1} f_{1} \mu_{t} \frac{\varepsilon}{k} P-c_{2} f_{2} \rho \frac{\varepsilon^{2}}{k}
\end{array}\right)
$$

where $P$ represents the production of kinetic energy and the following form is used:

$$
P=\left(\mu_{t}\left(\frac{\partial u_{i}}{\partial x_{j}}+\frac{\partial u_{j}}{\partial x_{i}}-\frac{2}{3} \frac{\partial u_{k}}{\partial x_{k}} \delta_{i j}\right)-\frac{2}{3} \rho k \delta_{i j}\right) \frac{\partial u_{i}}{\partial x_{j}}
$$




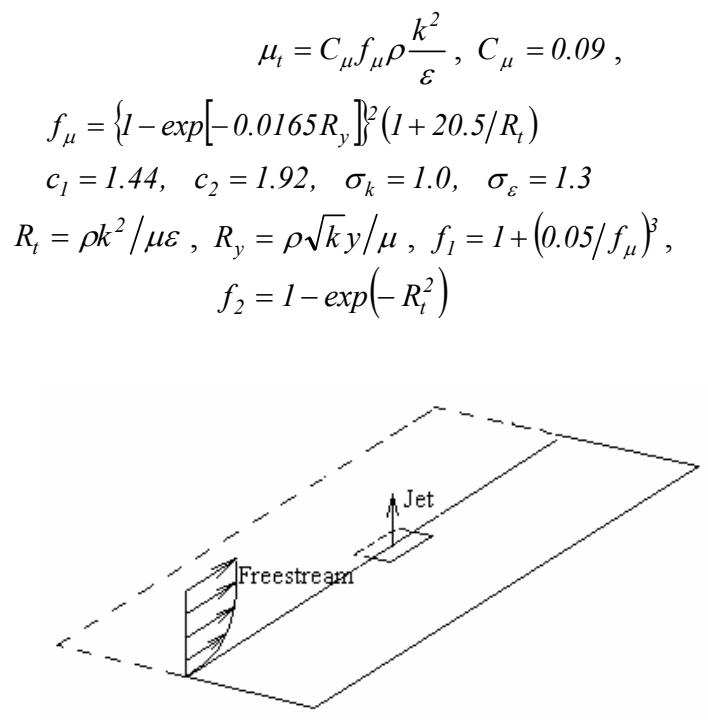

Figure 2: A flow field around an injected jet.

\section{Numerical method}

\subsection{Spatial discretization}

The semi-discrete form of Eq. (1) can be written as:

$$
\begin{aligned}
\frac{\partial Q}{\partial t} & =-\left[\left(E-E_{v}\right)_{i+1 / 2, j, k}-\left(E-E_{v}\right)_{i-l / 2, j, k}\right] \\
& -\left[\left(F-F_{v}\right)_{i, j+1 / 2, k}-\left(F-F_{v}\right)_{i, j-l / 2, k}\right] \\
& -\left[\left(G-G_{v}\right)_{i, j, k+1 / 2}-\left(G-G_{v}\right)_{i, j, k-1 / 2}\right]
\end{aligned}
$$

The spatial differencing of numerical fluxes adopts the fifth-order accurate WENO scheme of Jiang and Shu [6] for the inviscid convective fluxes and the fourth-order central differencing for the viscous fluxes.

The key idea of the WENO is to use a combination of all the candidate stencils to approximate the fluxes at the boundaries to a higher-order accuracy and at the same time to avoid spurious numerical oscillations near shocks instead of using only one of the candidate stencils.

By adopting the WENO scheme, we split the physical fluxes (say, $\hat{F}$ ) locally into positive and negative parts as

$$
\hat{F}(\hat{Q})=\hat{F}^{+}(\hat{Q})+\hat{F}^{-}(\hat{Q})
$$

where $\partial \hat{F}^{+} / \partial \hat{Q} \geq 0$ and $\partial \hat{F}^{-} / \partial \hat{Q} \leq 0$. In this paper, the local Lax-Friedrichs flux splitting method is used.

$$
\hat{F}^{ \pm}(\hat{Q})=\frac{1}{2}(\hat{F}(\hat{Q}) \pm|\Lambda| \hat{Q})
$$


where $|\Lambda|=\operatorname{diag}\left(\left|\lambda_{1}\right|,\left|\lambda_{2}\right|, \quad\left|\lambda_{3}\right|,\left|\lambda_{4}\right|,\left|\lambda_{5}\right|\right)$, and $\lambda_{1}, \lambda_{2}, \lambda_{3}, \lambda_{4}, \lambda_{5}$ are the local eigenvalues. We first consider the one-dimensional scalar conservation laws. For example,

$$
u_{t}+f(u)_{x}=0
$$

Let us discretize the space into the uniform interval of size $\Delta x$ and denote $x_{j}=j \Delta x$. The spatial operator of the WENO schemes, which approximates $-f(u)_{x}$ at $x_{j}$, will take the conservative form

$$
L=-\frac{1}{\Delta x}\left(\tilde{f}_{j+1 / 2}-\tilde{f}_{j-1 / 2}\right)
$$

where $\tilde{f}_{j+1 / 2}$ and $\tilde{f}_{j-1 / 2}$ are numerical fluxes. Designate $\tilde{f}_{j+1 / 2}^{+}$and $\tilde{f}_{j+1 / 2}^{-}$, respectively, the positive and negative parts of numerical flux, $\tilde{f}_{j+1 / 2}$, we have

$$
\tilde{f}_{j+1 / 2}=\tilde{f}_{j+1 / 2}^{+}+\tilde{f}_{j+1 / 2}^{-}
$$

Here we only describe how to compute $\tilde{f}_{j+1 / 2}^{+}$on the basis of the WENO. $\tilde{f}_{j+1 / 2}^{+}$can be shown symmetrically as follows:

$$
\begin{aligned}
\tilde{f}_{j+1 / 2}^{+} & =\omega_{0}^{+}\left(\frac{2}{6} f_{j-2}^{+}-\frac{7}{6} f_{j-1}^{+}+\frac{11}{6} f_{j}^{+}\right) \\
& +\omega_{1}^{+}\left(-\frac{1}{6} f_{j-1}^{+}+\frac{5}{6} f_{j}^{+}+\frac{2}{6} f_{j+1}^{+}\right) \\
& +\omega_{2}^{+}\left(\frac{2}{6} f_{j}^{+}+\frac{5}{6} f_{j+1}^{+}-\frac{1}{6} f_{j+2}^{+}\right)
\end{aligned}
$$

where

$$
\begin{gathered}
\omega_{k}^{+}=\frac{\alpha_{k}^{+}}{\alpha_{0}^{+}+\alpha_{1}^{+}+\alpha_{2}^{+}}, \quad k=0,1,2 \\
\alpha_{0}^{+}=\frac{1}{10}\left(\varepsilon+I S_{0}^{+}\right)^{-2}, \quad \alpha_{1}^{+}=\frac{6}{10}\left(\varepsilon+I S_{1}^{+}\right)^{-2} \quad \alpha_{2}^{+}=\frac{3}{10}\left(\varepsilon+I S_{2}^{+}\right)^{-2}
\end{gathered}
$$

and

$$
\begin{aligned}
& I S_{0}^{+}=\frac{13}{12}\left(f_{j-2}^{+}-2 f_{j-1}^{+}+f_{j}^{+}\right)^{2}+\frac{1}{4}\left(f_{j-2}^{+}-4 f_{j-1}^{+}+3 f_{j}^{+}\right)^{2} \\
& I S_{I}^{+}=\frac{13}{12}\left(f_{j-1}^{+}-2 f_{j}^{+}+f_{j+1}^{+}\right)^{2}+\frac{1}{4}\left(f_{j-1}^{+}-f_{j+1}^{+}\right)^{2} \\
& I S_{2}^{+}=\frac{13}{12}\left(f_{j}^{+}-2 f_{j+1}^{+}+f_{j+2}^{+}\right)^{2}+\frac{1}{4}\left(3 f_{j}^{+}-4 f_{j+1}^{+}+f_{j+2}^{+}\right)^{2}
\end{aligned}
$$

Equation (13) represents the smoothness measurement of stencils. Through the smoothness measurement, the interpolation polynomial on each stencil is assigned a weight from which we can construct a polynomial to approximate the numerical fluxes by combining all the polynomials. 


\subsection{Time discretization}

The time discretization of the WENO scheme can be implemented by the third-order Runge-Kutta method. To solve the ordinary differential equation

$$
\frac{d u}{d t}=L(u)
$$

where $L$ is a discretization of the spatial operator, the third-order Runge-Kutta is simply

$$
\begin{aligned}
& u^{(I)}=u^{n}+\Delta t L\left(u^{n}\right) \\
& u^{(2)}=\frac{3}{4} u^{n}+\frac{1}{4} u^{(1)}+\frac{1}{4} \Delta t L\left(u^{(I)}\right) \\
& u^{n+1}=\frac{1}{3} u^{n}+\frac{2}{3} u^{(2)}+\frac{2}{3} \Delta t L\left(u^{(2)}\right)
\end{aligned}
$$

\section{Presentation of results and discussion}

\subsection{Cross-flow over two-dimensional flat surface}

The computed flow field resulting from the transverse injection of two-dimensional sonic jets into a supersonic turbulent flow at a Mach number of 3.71 and a unit Reynolds number of $2.01 \times 10^{7}$ are simulated by employing the WENO scheme. The result is compared with the experiment and published calculations at the same condition [7].

The computational domain is $88 \mathrm{~mm} \times 50 \mathrm{~mm}$ with the slot width of $1 \mathrm{~mm}$. The free-stream Mach number is 3.71 and total pressure is 1atm. The jet is sonic and the total pressure is $0.31 \mathrm{~atm}$. The law of the wall coordinates $y^{+}$for the first mesh point has been maintained as $y^{+}<1$ in all cases.

Mach number, density and pressure contours are shown in Figs. 3(a) and (b) for 3-D and 2-D, respectively. The upstream separation shock and the induced bow shock are clearly presented in Fig. 3. The jet normal shock and the recompression shock downstream are also captured. In this figure the presence of two recirculation zones in the upstream and a recirculation zone in the downstream sections are clearly demonstrated. The surface static pressure distributions are compared with the test data of Aso and Okuyama [7] in Fig. 4. The present computations are also compared with the simulations obtained by Toda and Yamamoto [2]. The agreement of the present computation is better that the prediction by Yamamoto along the upstream location due to the improved prediction method for the approaching flow field. However, in the downstream location, both computations seem to be nearly identical. There appear two peaks upstream of the jet that corresponds to the separation and the stagnation line between the two counter rotating vortices upstream. The pressure peak in the downstream flowfield corresponds to the reattachment. It shows excellent agreement. 

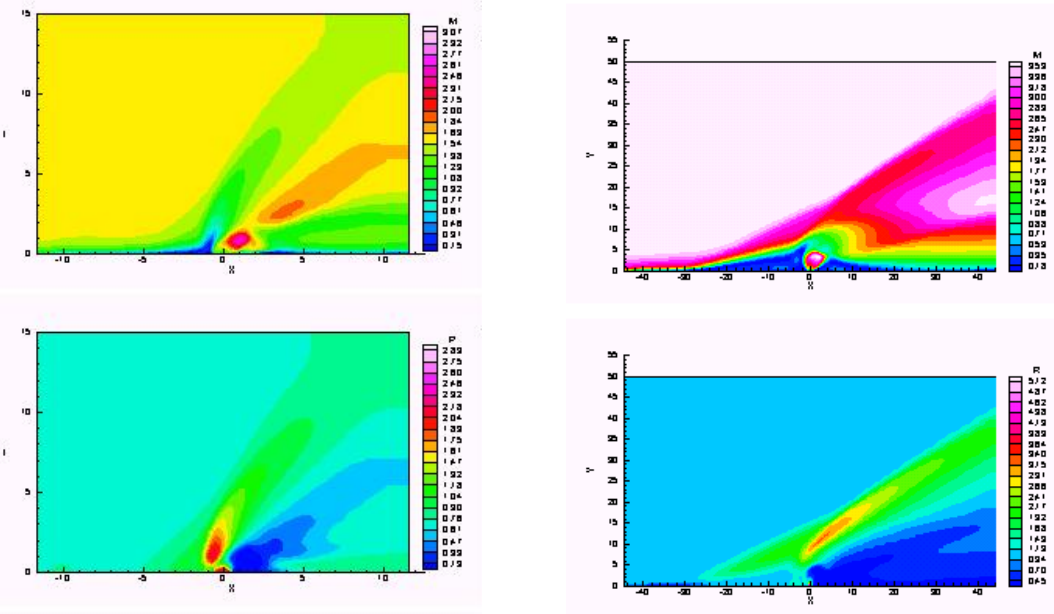

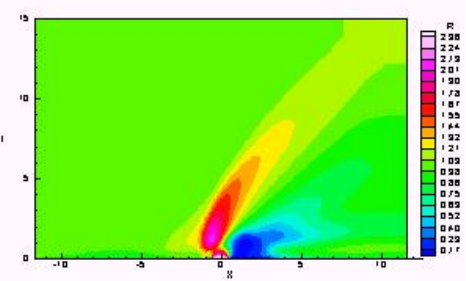

(a)

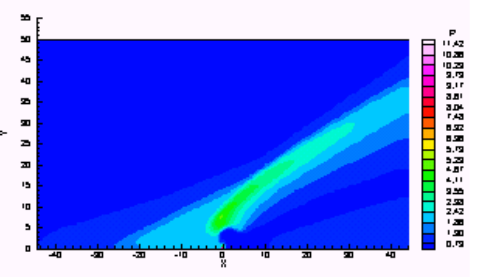

(b)

Figure 3: $\quad$ Mach number, density and pressure distributions. (a) 3-D, (b) 2-D.

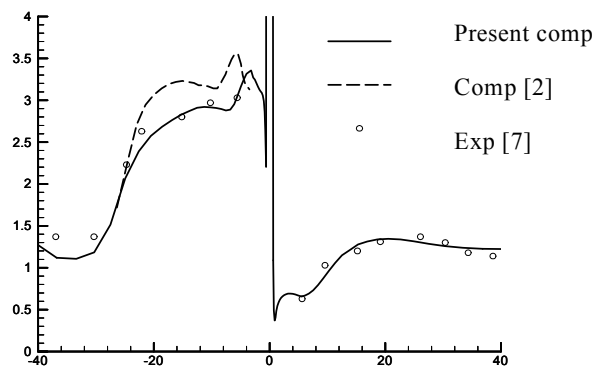

Figure 4: $\quad$ Pressure distribution along the plate.

\subsection{Three-dimensional results}

The configuration is a square hole in a flat plate. The computational domain is $37 \mathrm{~mm}$ long, $22 \mathrm{~mm}$ wide, and $25 \mathrm{~mm}$ high. There are 69 points in the streamwise direction and $59 \times 51$ in a cross plane. 
Free-stream Mach number is 3.0 and total pressure is 1atm. Jet is sonic and total pressure is $0.5 \mathrm{~atm}$.

Boundary conditions are: supersonic inlet, supersonic extrapolated exit, extrapolated upper boundary, and adiabatic no slip on the flat plate. The jet exit is set with uniform conditions and oriented normal to the surface.

Velocity vector plots and Mach contours in symmetrical plane are shown in Figs. 5 and 6, respectively. An induced bow shock is clearly presented. From the Mach number contour plot, the barrel shock and Mach disk are presented. As the jet is injected, it expands through a Prandtl-Meyer fan centered at the nozzle lip, compresses through the barrel shock, and then passes through a Mach disk.

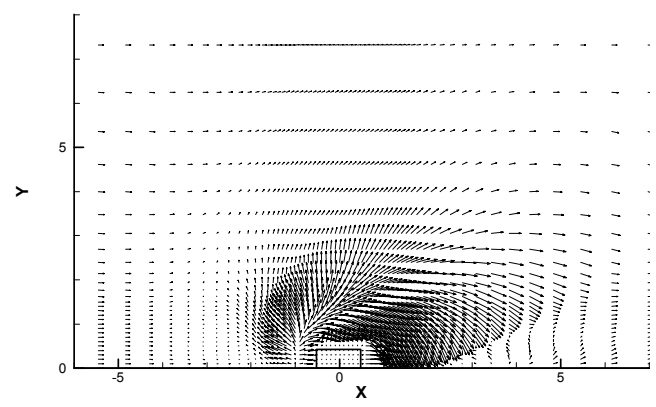

Figure 5: Velocity vectors in symmetrical section.

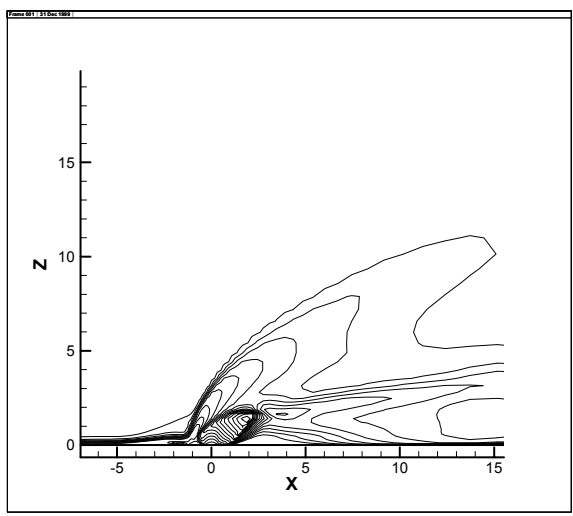

Figure 6: Mach contours in symmetrical section.

Figure 7 presents the wall pressure distribution in symmetrical plane. There are two main vortices in upstream of the jet, which are called the primary vortex and the secondary vortex. The primary vortex is induced by the boundary layer separation whereas the secondary vortex is induced by the injection. Furthermore, between the primary vortex and the wall, another vortex is shown, 
which is called the tertiary vortex. In the symmetrical plane, the wall pressure jumps up with the boundary layer separation and in the stagnation zone between the primary and the secondary vortices, which form the second pressure peak.

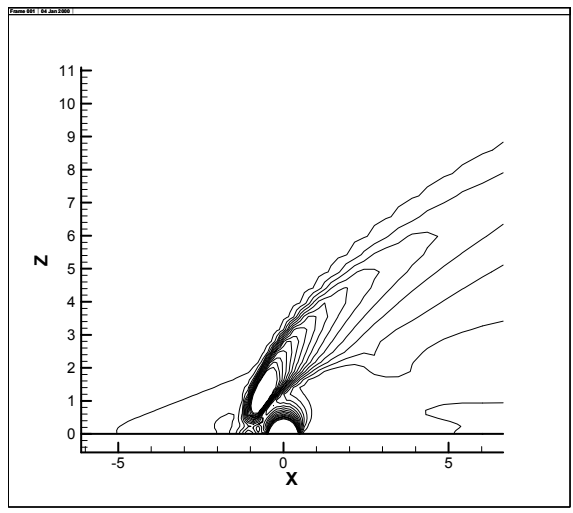

Figure 7: Wall pressure distribution.

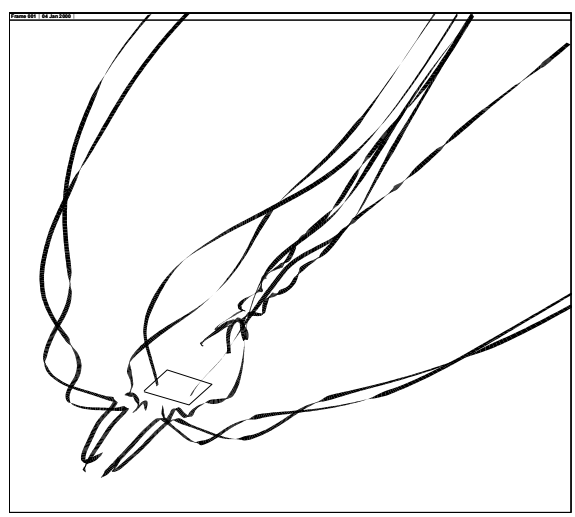

Figure 8: $\quad$ Streamlines around the jet.

Figure 8 presents the vortex structure by streamlines. There are four pairs of vortices in the flowfield. Horseshoe vortices form in the near-wall region from the vorticity within the cross-flow boundary layer and the vorticity is generated due to the wall pressure gradient resulting from the jet/freestream interaction. The shear layer vortices are developed from the vorticity contained in the jet boundary layer, and roll up into the free-stream. The counter-rotating structures are also formed from the vorticity presented in the jet boundary layer, and oriented in the streamwise direction. In this paper, other vortices are observed in the wake region downstream of the injector, which is called the helical vortex. 
Figure 9 presents the vortex structure by streamlines. There are four pairs of vortices in the flowfield. Horseshoe vortices form in the near-wall region from the vorticity within the cross-flow boundary layer and the vortices is generated due to the wall pressure gradient resulting from the jet/freestream interaction. The shear layer vortices are developed from the vorticity contained in the jet boundary layer, and roll up into the free-stream. The counter-rotating structures are also formed from the vorticity presented in the jet boundary layer, and oriented in the streamwise direction. In this paper, other vortices are observed in the wake region downstream of the injector, which is called the helical vortex (see Fig.10).

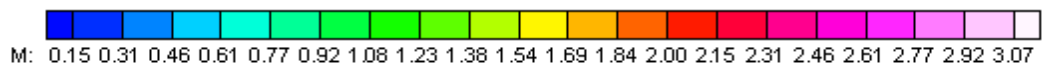

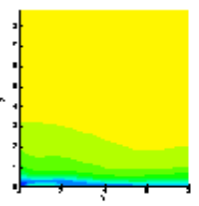

$\mathrm{X}=-1.5$

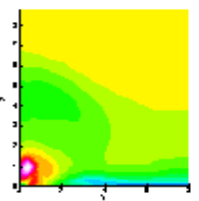

$\mathrm{x}=1.0$

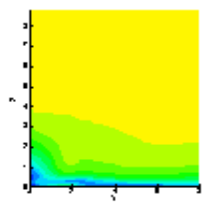

$\mathrm{X}=-1.0$

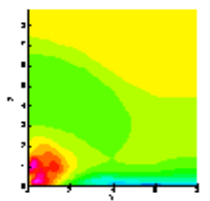

$\mathrm{X}=1.5$

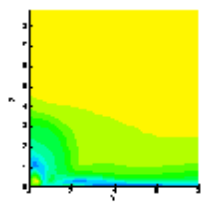

$\mathrm{x}=-0.5$

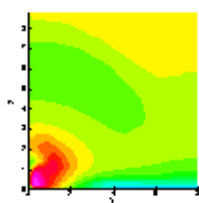

$\mathrm{X}=2.275$

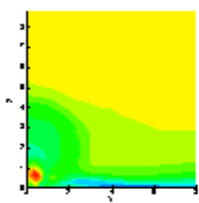

$\mathrm{x}=0.0$

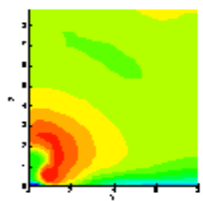

$\mathrm{X}=3.835$

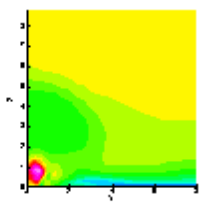

$\mathrm{X}=0.5$

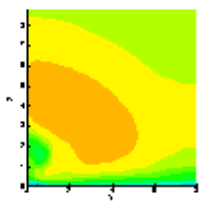

$\mathrm{x}=6.972$

Figure 9: $\quad$ Mach number contour at different stream locations.

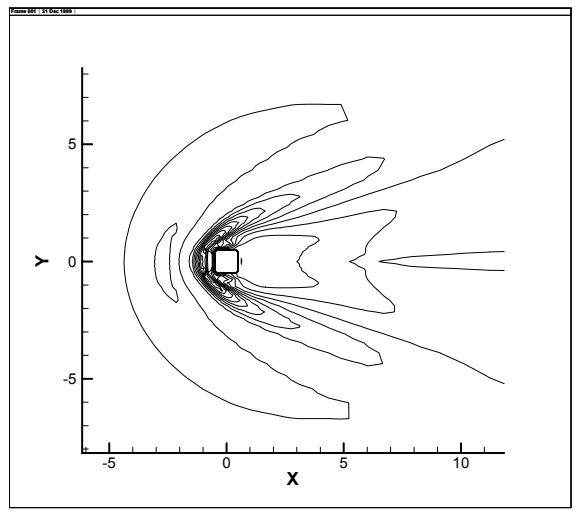

Figure 10: Surface pressure contours around jet. 


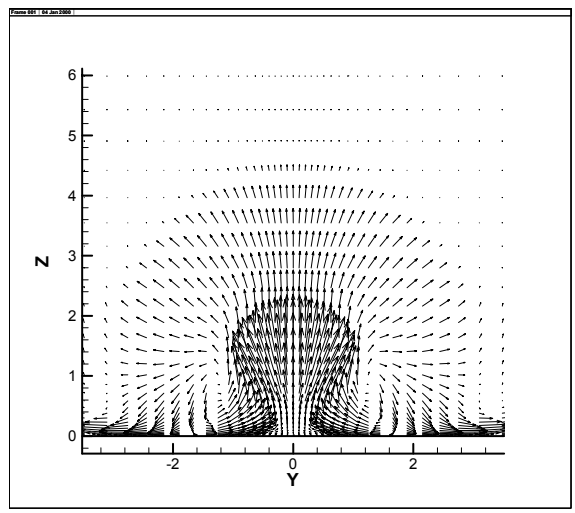

Figure 11: Velocity vectors in transverse section downstream of jet.

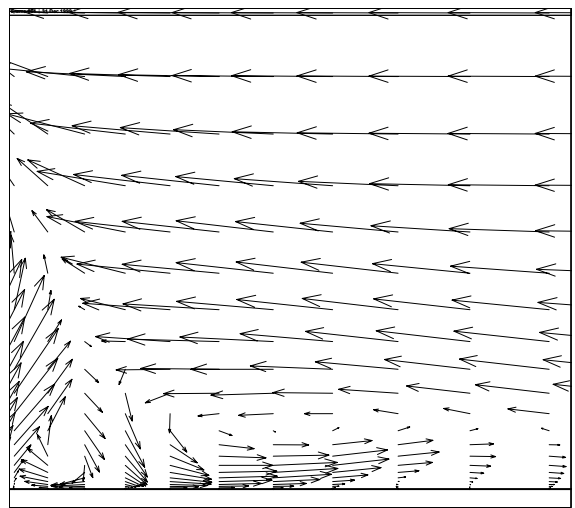

Figure 12: Velocity vectors upstream of jet.

Figure 11 presents the velocity vectors in cross-section where the helical vortices exist.

The primary vortex is induced by the boundary layer separation whereas the secondary vortex is induced by the injection. Furthermore, between the primary vortex and the wall, another vortex is shown, which is called the tertiary vortex. As shown in Fig. 12, in the symmetrical plane, the wall pressure jumps up with the boundary layer separation and in the stagnation zone between the primary and the secondary vortices, which form the second pressure peak. 


\section{Conclusion}

Applications of the WENO scheme and the low-Reynolds number $k$ - $\varepsilon$ turbulence model can accurately simulate the supersonic flow field with a transverse injection.

As the flow passed around the injector port, the boundary layer in front of the jet was separated and the bow shock was formed. In front of the jet, a boundary layer separation formed horseshoe vortices. Counter-rotating vortices were formed from the vorticity presented in the jet boundary layer, and oriented in the stream-wise direction. In the wake region downstream of the injector, helical vortices were observed.

\section{Acknowledgment}

The computations were performed in ORIGIN2000 at National Center for Supercomputing Applications under NSF grant CTS000003N.

\section{References}

[1] Gruber, M. R. and Goss, L. P., "Surface Pressure Measurements in Supersonic Transverse Injection Flowfields," Journal of Propulsion and Power, Vol. 15, No. 5, September-October 1999.

[2] Toda, K. and Yamamoto, M. "Computation of Supersonic Turbulent Flowfield with Secondary Jet normal to Freestream," AIAA 98-0944, 1998.

[3] Roger, R. P. and Chan, S. C., "Parameters Affecting Penetration of a Single Jet into a Supersonic Crossflow," AIAA 98-0425, 1998.

[4] Harten, A., Engquist, B, Osher, S., and Chakravarthy, "Uniformly high-order accurate nonoscillatory schemes," J. Comp. Phys. 71, 231, 1987.

[5] Wilcoxson, M. and Manousiouthakis, Vasilioss, "On an Implicit ENO Scheme," J. Comp. Phys. 115, 376-389, 1994.

[6] Jiang, Guang-Shan and Shu, Chi-Wang, "Efficient Implementation of Weighted ENO Schemes," J. Comp. Phys. 126, 202-228, 1996.

[7] Aso, S. and Okuyama, S., "Experimental Study on Mixing Phenomena in Supersonic Flows with Slot Injection”, AIAA Paper 91-0016, January 1991. 